\title{
Therapeutic antisense oligonucleotides against cancer: hurdling to the clinic
}

\author{
Pedro M. D. Moreno ${ }^{1 *}$ and Ana P. Pêgo ${ }^{1,2,3}$ \\ ${ }^{1}$ Instituto de Engenharia Biomédica, Nanobiomaterials for Targeted Therapies Group, Porto, Portugal \\ ${ }^{2}$ Faculdade de Engenharia, Universidade do Porto, Porto, Portugal \\ ${ }^{3}$ Instituto de Ciências Biomédicas Abel Salazar, Universidade do Porto, Porto, Portugal
}

Edited by:

João Conde, Massachusetts

Institute of Technology, USA

Reviewed by:

Juewen Liu, University of Waterloo,

Canada

Ramon Eritja, Institut de Química

Avançada de Catalunya - Consejo

Superior de Investigaciones

Cientificas, Spain

${ }^{*}$ Correspondence:

Pedro M. D. Moreno, Instituto de

Engenharia Biomédica,

Nanobiomaterials for Targeted

Therapies Group, Rua do Campo

Alegre, 823, Porto, Portugal

e-mail:pedro.moreno@ineb.up.pt
Under clinical development since the early 90's and with two successfully approved drugs (Fomivirsen and Mipomersen), oligonucleotide-based therapeutics has not yet delivered a clinical drug to the market in the cancer field. Whilst many pre-clinical data has been generated, a lack of understanding still exists on how to efficiently tackle all the different challenges presented for cancer targeting in a clinical setting. Namely, effective drug vectorization, careful choice of target gene or synergistic multi-gene targeting are surely decisive, while caution must be exerted to avoid potential toxic, often misleading off-target-effects. Here a brief overview will be given on the nucleic acid chemistry advances that established oligonucleotide technologies as a promising therapeutic alternative and ongoing cancer related clinical trials. Special attention will be given toward a perspective on the hurdles encountered specifically in the cancer field by this class of therapeutic oligonucleotides and a view on possible avenues for success is presented, with particular focus on the contribution from nanotechnology to the field.

Keywords: antisense, oligonucleotides, cancer, therapeutics, nanomedicine

\section{OPENING THE THERAPEUTIC LANDSCAPE BY EVOLUTION OF NUCLEIC ACIDS CHEMISTRY}

Oligonucleotides have been under investigation for over 30 years, whilst achieving only two approved drugs. Those were, Fomivirsen, approved by the FDA in 1998 for the treatment of cytomegalovirus retinitis in patients with AIDS, but discontinued for low demand, and Mipomersen, FDA approved in 2013, targeting ApoB100 for the treatment of homozygous familial hypercholesterolaemia $(\mathrm{HoFH})$, a rare genetic disorder that leads to excessive levels of low-density lipoprotein (LDL) cholesterol. These are both single-stranded antisense oligonucleotide drugs (most commonly known as AONs) that together with siRNA (a double-stranded oligonucleotide) make up, at present, the therapeutic antisense oligonucleotide field. In this paper more emphasis will be put on AONs due to their longer time in development and history of clinical trials.

Progress in this field has been proceeding at a steady but somewhat slow pace, driven mostly by the speed at which the different intra and extracellular obstacles encountered by the oligonucleotide drugs are being tackled. The most important hurdles have been (i) the poor stability against extra- and intracellular degradation (mostly by action of nucleases), (ii) inefficient intracellular delivery to target cells or tissues, (iii) inadequate affinity toward the intended target sequence and (iv) potential off-target/toxicity effects. Finally for most applications (v) immunostimulation has also been a matter of concern.

The pursuit of clinically relevant antisense drugs has led the field to develop different types of chemical modifications to native DNA or RNA in an attempt to overcome the aforementioned limitations. Most widely used modifications can be divided in two simple categories: (a) backbone structure and (b) sugar ring modifications (Table 1).

The main goals of these chemical modifications have been to achieve increased resistance to degradation by exo- and endonucleases; increase affinity, and in some cases selectivity, toward target RNA/DNA sequences and to modulate the immunostimulation properties of the oligonucleotides.

In the case of phosphorothioate (PS) modification (one of the first and widely used modifications introduced in therapeutic antisense oligonucleotides) (Eckstein, 1967), it has also led to better pharmacokinetics and extended circulation times for AONs systemically applied in a "naked" form (i.e., unprotected by delivery agents). This effect has been attributed to unspecific binding to serum proteins such as albumin (Srinivasan et al., 1995; Crooke et al., 1996; Watanabe et al., 2006).

Mechanistically, AONs work via binding to a specific RNA target sequence resulting in the block of RNA function. This can be achieved through steric hindrance (non-degradative pathway) and concomitant RNA translation block, or target degradation. The latter occurs by the action of an endogenous enzyme, RNase $\mathrm{H}$, or alternatively, by a catalytic cleavage activity embedded into the oligonucleotide itself (e.g., ribozymes and DNAzymes) (Bennett and Swayze, 2010). Notably, the non-degradative mechanism, through steric hindrance, has recently been exploited, with great success, for modulation of pre-mRNA splice patterns by affecting the binding of trans-splicing regulatory factors to the pre-mRNA (Hammond and Wood, 2011; Bestas et al., 2014; Disterer et al., 2014). 
Table 1 | Common nucleic acids modifications divided by category.
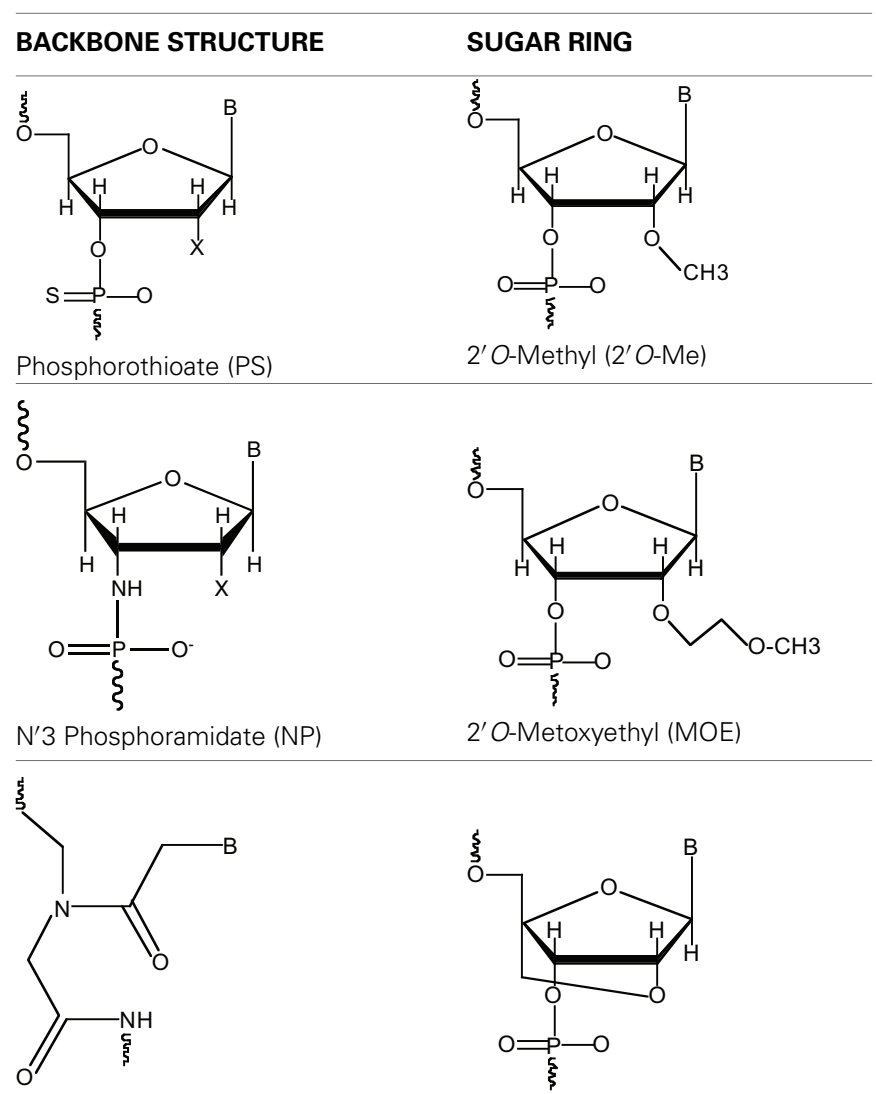

Peptide Nucleic Acid (PNA)

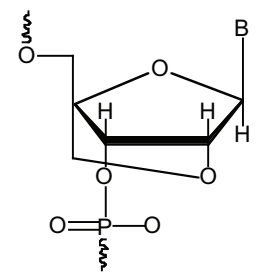

Locked Nucleic Acid (LNA)
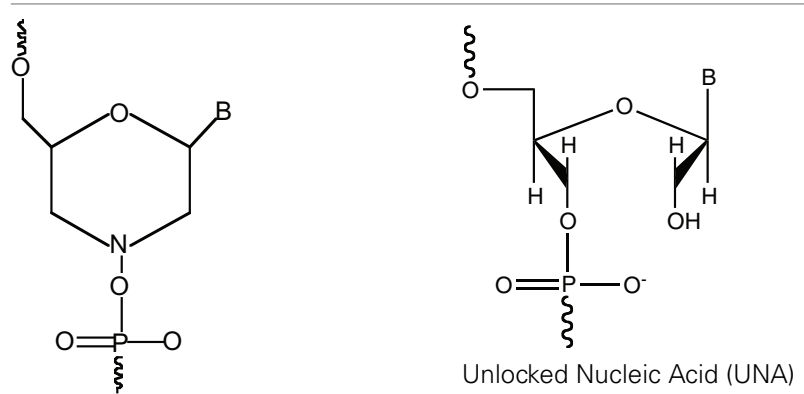

Morpholino (PMO)

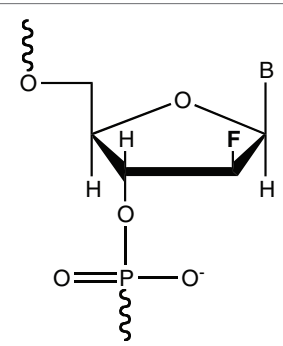

2' F-Arabino Nucleic Acid (2' F-ANA)

The mechanism of action of AONs has to be carefully considered when deciding for the type of nucleotide modifications and design of the oligonucleotide (here including number and position of modified nucleotides). Thus, in contrast to PS modifications that maintain the anionic character of oligonucleotides, PNAs and PMOs completely substitute the phosphodiester linkage by either a polyamide backbone (Nielsen et al., 1991) or a phosphorodiamidate group (Summerton and Weller, 1997), respectively, hence being uncharged nucleotide analogs. On the other hand sugar ring modifications can influence the nucleoside conformation promoting the preferential adoption of an A-form (dsRNA type) or B-form (dsDNA type) helix when in a doublestranded structure. In the case of $2^{\prime} \mathrm{O}-\mathrm{Me}$ RNA (Kawai et al., 1992; Nishizaki et al., 1997), MOE (Manoharan, 1999) and LNA (Koshkin et al., 1998; Obika et al., 1998), all promote the A-form while $2^{\prime} F$-ANA (Berger et al., 1998) the B-form. Contrasting all the aforementioned, UNA, with its unlocked ring configuration, does not impart any conformation restrictions (Pasternak and Wengel, 2011).

All of the abovementioned nucleotide modifications have thus been used with few restrictions when designing steric hindrance AONs, since their incorporation mainly focus on achieving enhanced binding affinity and selectivity toward a target sequence. In contrast, the design of AONs for target degradation through the action of RNAse $\mathrm{H}$ has to obey the enzyme's structural preferences for cleaving DNA/RNA duplexes (Minshull and Hunt, 1986; Nakamura et al., 1991). Hence, all modifications too divergent from natural DNA nucleotides need to be carefully considered to not hinder the enzyme action. This can be accomplished by the design of "gapmer" AONs containing the modified nucleotides on the $5^{\prime}$ and $3^{\prime}$ terminus flanking a central unmodified DNA nucleotides stretch (Monia et al., 1993; Stanton et al., 2012). Specifically, in the case of the two approved drugs, Fomivirsen is a PS modified DNA oligonucleotide applied by intraocular injection, whereas Mipomersen is a second generation AON gapmer with MOE modifications at the ends and PS throughout, applied as an intravascular injection.

\section{BRIEF OVERVIEW ON ANTISENSE OLIGONUCLEOTIDES CLINICAL TRIALS RELATED TO CANCER}

An increasing number of clinical trials with AONs are ongoing, which shows that the field is rapidly forwarding. In Table 2 a list of recently completed and on-going clinical trials is presented.

Other studies have unfortunately failed, in different phases, to reach their expected endpoints or to show significant benefit, leading to a stop in the corresponding AON development. Some aspects of antisense technology have contributed to this and are next discussed.

\section{CHALLENGES FOR ANTISENSE TECHNOLOGY-1. UNSPECIFIC MODES OF ACTION}

Along their development path, oligonucleotides have unraveled much of their potential but also many of their limitations.

As discussed above, introduction of PS modifications led to the first evidences that antisense drugs could become a reality in a clinical setting, essentially by increasing resistance to degradation and extending circulation times after systemic administration (mostly due to unspecific serum-protein binding). These properties improved the oligonucleotide therapeutic potential, despite some decreased affinity for the target sequence (when comparing to regular DNA oligonucleotides) (Kibler-Herzog et al., 1991). On 
Table 2 | On-going and recently completed anti-cancer AON clinical trials.

\begin{tabular}{|c|c|c|c|c|c|}
\hline DRUG & AON (carrier) & TARGET & INDICATION & STATUS & DEVELOPER \\
\hline $\begin{array}{l}\text { EGFR antisense } \\
\text { DNA }\end{array}$ & $\begin{array}{l}\text { Phoshorothioate ODN } \\
\text { ("naked") }\end{array}$ & EGFR & $\begin{array}{l}\text { Advanced Head and Neck } \\
\text { Squamous Cell Carcinoma }\end{array}$ & Phase I/II (recruiting) & University of Texas \\
\hline $\begin{array}{l}\text { ISIS-STAT3Rx (ISIS } \\
\text { 481464/ AZD9150) }\end{array}$ & $\begin{array}{l}\text { cEt-PS gapmer ODN } \\
\text { ("naked") }\end{array}$ & STAT3 & $\begin{array}{l}\text { Lymphoma; hepatocellular } \\
\text { carcinoma }\end{array}$ & Phase I/II (recruiting) & $\begin{array}{l}\text { Astrazeneca } \\
\text { (ISIS Pharmaceuticals) }\end{array}$ \\
\hline EZN-2968 & $\begin{array}{l}\text { LNA-PS gapmer ODN } \\
\text { ("naked") }\end{array}$ & $\mathrm{HIF}-1 \alpha$ & Advanced solid tumors & Phase I (completed) & $\begin{array}{l}\text { Enzon Pharmaceuticals } \\
\text { (Santaris Pharma) }\end{array}$ \\
\hline LErafAON-ETU & $\begin{array}{l}\text { DNA-PS modified at } 5^{\prime} \text { and } 3^{\prime} \text { end } \\
\text { (lipossome) }\end{array}$ & c-raf & Advanced Cancer & $\begin{array}{l}\text { Phase I } \\
\text { (completed) }\end{array}$ & INSYS Therapeutics Inc \\
\hline
\end{tabular}

ODN-oligodeoxynucleotide; cEt_constrained Ethyl.

the other hand, this unspecific protein binding feature can potentially lead to associated toxicities or cellular effects not entirely sequence specific, such as complement activation, increased coagulation times and unwanted immune activation (Brown et al., 1994; Krieg and Stein, 1995; Henry et al., 1999; Mou et al., 2001; Krieg et al., 2003; Senn et al., 2005). These effects, however, are most often oligonucleotide length and concentration dependent (Webb et al., 2001). Immune activation, on the other hand, is also enhanced by specific nucleotide sequences (CpG motifs) (Barchet et al., 2008), although this can be minimized by different types of nucleotide modifications (Henry et al., 2000). Nevertheless, immune activation is an important factor that has previously led to erroneous interpretations of data when inhibition of tumor growth was not primarily driven by the antisense mechanism but by the immunostimulatory properties of $\mathrm{CpG}$ sequences found in certain AONs (Badros et al., 2005; Gekeler et al., 2006). Regarding potential PS-derived unspecific cellular effects these have been proposed to affect the mechanism of action of an anti-cancer oligonucleotide drug by the down-regulation of several anti-apoptotic proteins and glycolytic enzymes. These were actually seen as important contributors to the apoptotic action (Stessl et al., 2009; Winkler et al., 2010).

Another important concern relates to hybridization dependent toxicity, deriving from exaggerated pharmacological action (a consequence also seen with any other chemical drug), or offtarget hybridization. The latter can be minimized by designing the antisense drug taking into account a detailed bioinformatics analysis for identification of both, genes with perfect matches or with partial complementarity (looking out for 1-3 mismatches as the most relevant ones) (Bennett and Swayze, 2010).

The above considerations have raised some difficulties, especially in vivo, for the exact prediction of the mechanism of action of an antisense drug and are among the causes probably hampering a more resolute demonstration of the therapeutic relevance of antisense drugs toward not only cancer but also other diseases in general. This concern can be demonstrated by the case of the antisense drug LY2275796 (a second generation AON with PS and MOE modifications targeting eIF-4E) where, besides target gene downregulation, housekeeping genes were considerably affected as well, raising the question to whether the antisense action was sequence specific or also mediated by off-target effects (Hong et al., 2011).

This scenario only reinforces the need for an in-depth pharmacologic and pharmacokinetic analysis at the preclinical stage of AON development.

\section{CHALLENGES FOR ANTISENSE TECHNOLOGY-2. DELIVERY}

The efficient and targeted delivery of nucleic acid therapeutics is seen as, if not the biggest, one of the most important challenges for this class of drugs. The most commonly used nucleic acids drugs (namely, plasmid DNA, siRNA and AONs) have specific features influencing their cellular uptake and delivery vector development. AONs, due to the short chain size have very low charge density, in addition, being single-stranded, they have the 
aromatic bases exposed (not buried inside a double helix), which confers a slight hydrophobic character to the molecule. These properties, enable some level of interaction with the cell membrane, which can be further potentiated by PS modifications, making possible, although still extremely inefficient, their use without the help of any vector formulations (Watts and Corey, 2012). This has been recently emphasized by the demonstration that short LNA modified AONs were able to sustain gene downregulation in a large variety of cell lines when administered in vitro unassisted by transfection agents (also referred as gymnotic delivery), although some cell lines still seem to be completely refractory to this type of AON uptake (Stein et al., 2010). The results obtained by gymnotic delivery seem to correlate well with the obtained in vivo gene silencing efficiencies for the "naked" LNA administration; in fact, a better prediction of in vivo potency was obtained in comparison to data resulting from transfection-mediated in vitro AON delivery, a more standard method to preliminarily analyze AON efficiency (Stein et al., 2010). A similar study showed downregulation of different cancer gene targets, by the gymnotic delivery of LNA-AON in over 30 cell lines, although discrepancies between both studies are seen when relating intracellular localization of the AONs (nuclear vs. cytoplasmatic) and efficient down-regulation activity (Zhang et al., 2011).

Despite several studies demonstrating some activity when using "naked" AONs in vivo, and their wide tissue distribution, it has also been realized that these preferentially accumulate in the liver and kidney and to a lesser extent in spleen, lymph nodes and bone marrow (Agrawal et al., 1995; Iversen et al., 1995; Graham et al., 1998; Geary, 2009; Straarup et al., 2010). Liver, as a primary location of oligonucleotide accumulation has received a greater level of attention with some of the most promising AON trials taking advantage of this effect, as seen with Mipomersen (Hovingh et al., 2013). Liver accumulation has been attributed to the role of this organ in clearance by the reticulum endothelium system (RES). This results from the abundant presence of phagocytic Kupffer cells, together with the high blood flow received and, importantly, the existence of a fenestrated vasculature with an average 100-200 $\mathrm{nm}$ pore diameter between endothelial lining cells (Wisse et al., 2008). It should be noted that the pharmacokinetics of AONs are dependent on chemistry, with the most favorable properties relating to the presence of PS linkages and the polyanionic character of the molecules. Thus, AONs based on PNA and PMO when administered as "naked" formulations in vivo, are rapidly cleared from circulation while showing poorer tissue distribution (Dirin and Winkler, 2013).

Tumor tissue also shares some of the abovementioned features, specially regarding its specific microvasculature characteristics (viz. for solid tumors). Fenestrations of 100-700 nm have been found in some tumor vessels, which together with a poor lymphatic drainage give rise to the enhanced permeability and retention effect (EPR) (Jang et al., 2003), responsible for the accumulation of macromolecules or nanoparticles in tumors. Another effect to consider is the usually high interstitial fluid pressure (IFP) in tumors that obviates the normal rapid convective flow from blood to the tissue interstitium (due to osmotic and hydrostatic pressure differences). This effect is counterproductive in terms of drug accessibility to the tumor tissue, which then has to rely in slow diffusion processes. A dense structure of interstitial matrix and cells also mounts a final barrier to the diffusion process (Chauhan et al., 2011). Finally, the uneven leakiness of vessels found in tumors further contributes to a highly heterogeneous process of drug penetration. Another consideration is that the larger the tumor the bigger the regional differences within the tumor itself. This is illustrated by the presence of a necrotic core with an almost complete absence of blood flow, a seminecrotic region with poor blood flow within un-branched vessels, a stable region with branched vessels and good flow and an active angiogenic front where blood flow is variable and can be substantially higher than in surrounding host normal tissues (Jain, 2012).

These hindrances can result in AONs despite reaching tumor tissue, not being able to accumulate to a significant extent in the tumor tissue, with the additional drawback of distributing unevenly throughout the tissue (Plenat et al., 1995; Delong et al., 1997; Devi et al., 2005).

Certainly these delivery issues hamper a more effective translation of anti-cancer antisense oligonucleotides to the clinic.

\section{PERSPECTIVES ON AON VECTORIZATION FOR CANCER THERAPEUTICS}

Given the wide tissue distribution properties of AONs and their preferential accumulation in organs other than tumor tissue, this can lead to the necessity of using high amounts of AONs in order to reach a meaningful biological effect, raising concerns due to presence of high AON concentrations in unspecific tissue/organs. In addition, although some level of localization to tumor tissue is attained due to the EPR effect, there can be a large heterogeneity in the targeting and distribution of AONs between tumors and within the same tumor. Not achieving a homogeneous and abundant distribution of AONs to the entire tumor can result in differential intracellular concentrations of the AON affecting functional efficiency and ultimately leading to some cells evading the anti-cancer action.

The development of nanocarriers for AON delivery could have a positive contribution in AON anti-cancer efficiency while minimizing toxicity, although their utility must be evaluated in a caseby-case basis. Nanoparticle systems will be also affected by inter and intra-tumor heterogeneity, where differences between tumor mass strongly influence the EPR and IFP effects. In fact, the EPR effect is more prevalent in tumors of $100 \mathrm{~mm}^{3}$ which limits its use when targeting small or unvascularized primary or secondary tumor (metastases) (Adiseshaiah et al., 2010). While AONs associated with nanoparticle systems can take greater advantage of the EPR effect, when "naked" administration is employed these will be affected to a wider extent by IFP similarly to small drugs Interestingly, this could mean that free AONs could have an advantage when dealing with a tumor with a less disturbed vascular architecture or when tumor vasculature normalization drugs are used (Juliano et al., 2009; Chauhan et al., 2012). This view can, however, be too simplistic as shown in a work dealing with imaging and modulation of $\mathrm{AON}$ microdistribution in solid tumor xenografts (Mocanu et al., 2007). It was seen that a drug-induced decrease in IFP was not accompanied by an expected improved distribution of the $\mathrm{AON}$, in contrast to what has been reported 
for some small drugs. This was attributed to a strong association of the AON with regions of necrosis/hypoxia or due to the effect of the drug promoting neovascularization and the less permeable status of the newly formed vessels. Also, one could reason that the tumor matrix and the specific collagen content along with the status of other fibrilliary proteins could affect distribution of AONs (Netti et al., 2000; Mocanu et al., 2007), especially when dealing with PS-AONs due to their unspecific binding properties. In contrast to tumor normalization, the EPR effect can be transiently augmented by modulation of blood pressure and local increase of blood flow through the use of angiotensin-II-induced hypertension and nitric oxide releasing agents (Fang et al., 2011). In this way uptake of nanoparticle systems could be favored.

In terms of available systems for vectorization of AONs these can be divided in nanoparticle systems formed by interactions of different carrier formulations with the AONs or nanoconjugates where $\mathrm{AONs}$ are covalently linked to different functional molecules (e.g., peptides, sugars) (Juliano et al., 2012; Yin et al., 2014).

Carrier formulations that have been frequently used for delivery of different nucleic acids comprise cationic lipids and polymers. The basic driving force of complex formation is the electrostatic interaction. In brief, the carrier system needs to (i) protect the nucleic-acid from extracellular and intracellular degradation, until it reaches its target, (ii) achieve a prolonged circulation time in order to be accumulated in the location of interest, (iii) efficiently interact with the cellular membrane to promote uptake (generally through endocytosis processes), (iv) promote escape from endocytic vesicles and finally (v) dissociate from the active nucleic-acid in order for it to function (Yin et al., 2014).

Cationic lipids generally used with nucleic acids (forming lipoplexes) comprise DOTMA, DOSPE, DOTAP, but also neutral lipids such as the fusogenic DOPE have been incorporated to improve transfection efficiency (Simoes et al., 2005). Some of these lipids have been studied specifically with AONs (Jaaskelainen et al., 2000; Meidan et al., 2001; Gokhale et al., 2002) but few have been utilized in pre-clinical or clinical work. A liposome formulation of c-raf antisense oligonucleotide constitutes the first example of an AON-lipoplex taken into clinical development stages (Zhang et al., 2009).

Polymers have been also used. These have an immense chemical diversity and are easy to chemically manipulate thus enabling tuning of properties by functionalization. Some examples of polymeric systems that have been utilized are poly(L-Lysine) (Stewart et al., 1996) and poly(ethylene imine) (Seong et al., 2006). However, some issues regarding efficiency and toxicity have warranted the development of other systems based on natural and biodegradable polymers such as chitosan (Gomes et al., 2014).

Also worth mentioning are delivery systems based on inorganic nanoparticles, an emerging field, of which, gold nanoparticles are perhaps the most representative ones (Ding et al., 2014). A detailed view on the intracellular transport (e.g., understanding its endocytic route) (Wu et al., 2014) and careful evaluation of toxicity profile (e.g., genotoxicity, membrane damage) (Alkilany and Murphy, 2010) can provide important information to the advancement of the technology into clinical development.
Taking into consideration the previously mentioned tumor features, some design specificities should be taken into account when implementing an AON-nanocomplex strategy as anticancer therapeutic platform. Regarding size, the smaller the particle the better the intra-tumoral transport $(<10 \mathrm{~nm})$, however the EPR effect will be less significant than for bigger particles (10$200 \mathrm{~nm}$ ) (Netti et al., 2000). On the other hand, particles on the higher size range will present a limited capacity to extravasate from vessel pores, but for the same reason will be more specific. Also, bigger sizes determine a higher clearance by the RES, although this can be counteracted by steric stabilization through poly(ethylene glycol) surface modification (Van Vlerken et al., 2007).

Surface charge also plays a crucial role. While cationic particles tend to target tumor endothelium and exhibit a higher vascular permeability than neutral or anionic ones, the fastest and more homogenously distribution in tumor interstitium is seen for the neutral particles. Presence of charge in particles contributes to aggregation with different components of the tumor matrix thus hindering transport. Accordingly, neutral or zwitterionic particles, or even particles with the property to change charge according to the microenvironment should perhaps be the best options (Chauhan et al., 2011). Shape, an often-overlooked property, likewise affects transport. Here factors such as rigidity and form (spherical vs. rod) come into play with flexible nanometer-sized particles showing, in principle, better transport characteristics (Chauhan et al., 2011).

In conclusion, the field of anti-cancer AONs is rapidly advancing, supported in part by the growing number of chemical modifications that conferred superior properties to AONs. However, specific and efficient delivery to tumors is still of uttermost importance. Uniform distribution throughout the tumor is an important challenge particularly due to intra-tumoral regional specificities and a progressive microenvironment. A further challenge lies in the dynamic nature of tumors that may correlate with temporal and spatial changes in expression of the AON target genes.

Multi-gene targeting AONs and efficient tumor targeting vectorization systems will, thus, be of uttermost importance in the development of a successful anti-cancer AON strategy.

\section{ACKNOWLEDGMENTS}

The authors would like to acknowledge the FEDER funds through the Programa Operacional Factores de CompetitividadeCOMPETE and the Portuguese funds through FCT—Fundação para a Ciência e a Tecnologia (PTDC/CTM-NAN/115124/2009, HMSP-ICT/0020/2010 and PEst-C/SAU/LA0002/2013) that supported this work. Pedro M. D. Moreno is supported by a Marie Curie Action of the European Community's Seventh Framework Program (PIEF-GA-2011-300485).

\section{REFERENCES}

Adiseshaiah, P. P., Hall, J. B., and McNeil, S. E. (2010). Nanomaterial standards for efficacy and toxicity assessment. Wiley Interdiscip. Rev. Nanomed. Nanobiotechnol. 2, 99-112. doi: 10.1002/wnan.66

Agrawal, S., Temsamani, J., Galbraith, W., and Tang, J. (1995). Pharmacokinetics of antisense oligonucleotides. Clin. Pharmacokinet. 28, 7-16. doi: 10.2165/00003088-199528010-00002 
Alkilany, A. M., and Murphy, C. J. (2010). Toxicity and cellular uptake of gold nanoparticles: what we have learned so far? J. Nanopart. Res. 12, 2313-2333. doi: 10.1007/s11051-010-9911-8

Badros, A. Z., Goloubeva, O., Rapoport, A. P., Ratterree, B., Gahres, N., Meisenberg, B., et al. (2005). Phase II study of G3139, a Bcl-2 antisense oligonucleotide, in combination with dexamethasone and thalidomide in relapsed multiple myeloma patients. J. Clin. Oncol. 23, 4089-4099. doi: 10.1200/JCO.2005.14.381

Barchet, W., Wimmenauer, V., Schlee, M., and Hartmann, G. (2008). Accessing the therapeutic potential of immunostimulatory nucleic acids. Curr. Opin. Immunol. 20, 389-395. doi: 10.1016/j.coi.2008.07.007

Bennett, C. F., and Swayze, E. E. (2010). RNA targeting therapeutics: molecular mechanisms of antisense oligonucleotides as a therapeutic platform. Annu. Rev. Pharmacol. Toxicol. 50, 259-293. doi: 10.1146/annurev.pharmtox. 010909.105654

Berger, I., Tereshko, V., Ikeda, H., Marquez, V. E., and Egli, M. (1998). Crystal structures of B-DNA with incorporated $2^{\prime}$-deoxy-2'-fluoro-arabino-furanosyl thymines: implications of conformational preorganization for duplex stability. Nucleic Acids Res. 26, 2473-2480. doi: 10.1093/nar/26.10.2473

Bestas, B., Moreno, P. M., Blomberg, K. E., Mohammad, D. K., Saleh, A. F., Sutlu, T., et al. (2014). Splice-correcting oligonucleotides restore BTK function in X-linked agammaglobulinemia model. J. Clin. Invest. 124, 4067-4081. doi: 10.1172/JCI76175

Brown, D. A., Kang, S. H., Gryaznov, S. M., Dedionisio, L., Heidenreich, O., Sullivan, S., et al. (1994). Effect of phosphorothioate modification of oligodeoxynucleotides on specific protein binding. J. Biol. Chem. 269, 26801-26805.

Chauhan, V. P., Stylianopoulos, T., Boucher, Y., and Jain, R. K. (2011). Delivery of molecular and nanoscale medicine to tumors: transport barriers and strategies. Annu. Rev. Chem. Biomol. Eng. 2, 281-298. doi: 10.1146/annurev-chembioeng061010-114300

Chauhan, V. P., Stylianopoulos, T., Martin, J. D., Popovic, Z., Chen, O., Kamoun, W. S., et al. (2012). Normalization of tumour blood vessels improves the delivery of nanomedicines in a size-dependent manner. Nat. Nanotechnol. 7, 383-388. doi: 10.1038/nnano.2012.45

Crooke, S. T., Graham, M. J., Zuckerman, J. E., Brooks, D., Conklin, B. S., Cummins, L. L., et al. (1996). Pharmacokinetic properties of several novel oligonucleotide analogs in mice. J. Pharmacol. Exp. Ther. 277, 923-937.

Delong, R. K., Nolting, A., Fisher, M., Chen, Q., Wickstrom, E., Kligshteyn, M., et al. (1997). Comparative pharmacokinetics, tissue distribution, and tumor accumulation of phosphorothioate, phosphorodithioate, and methylphosphonate oligonucleotides in nude mice. Antisense Nucleic Acid Drug Dev. 7, 71-77. doi: 10.1089/oli.1.1997.7.71

Devi, G. R., Beer, T. M., Corless, C. L., Arora, V., Weller, D. L., and Iversen, P. L. (2005). In vivo bioavailability and pharmacokinetics of a c-MYC antisense phosphorodiamidate morpholino oligomer, AVI-4126, in solid tumors. Clin. Cancer Res. 11, 3930-3938. doi: 10.1158/1078-0432.CCR-04-2091

Ding, Y., Jiang, Z., Saha, K., Kim, C. S., Kim, S. T., Landis, R. F., et al. (2014). Gold nanoparticles for nucleic acid delivery. Mol. Ther. 22, 1075-1083. doi: $10.1038 / \mathrm{mt} .2014 .30$

Dirin, M., and Winkler, J. (2013). Influence of diverse chemical modifications on the ADME characteristics and toxicology of antisense oligonucleotides. Expert Opin. Biol. Ther. 13, 875-888. doi: 10.1517/14712598.2013.774366

Disterer, P., Kryczka, A., Liu, Y., Badi, Y. E., Wong, J. J., Owen, J. S., et al. (2014). Development of therapeutic splice-switching oligonucleotides. Hum. Gene Ther. 25, 587-598. doi: 10.1089/hum.2013.234

Eckstein, F. (1967). A dinucleoside phosphorothioate. Tetrahedron Lett. 8, 1157-1160. doi: 10.1016/S0040-4039(00)90656-7

Fang, J., Nakamura, H., and Maeda, H. (2011). The EPR effect: unique features of tumor blood vessels for drug delivery, factors involved, and limitations and augmentation of the effect. Adv. Drug Deliv. Rev. 63, 136-151. doi: 10.1016/j.addr.2010.04.009

Geary, R. S. (2009). Antisense oligonucleotide pharmacokinetics and metabolism. Expert Opin. Drug Metab. Toxicol. 5, 381-391. doi: 10.1517/17425250902877680

Gekeler, V., Gimmnich, P., Hofmann, H. P., Grebe, C., Rommele, M., Leja, A., et al. (2006). G3139 and other CpG-containing immunostimulatory phosphorothioate oligodeoxynucleotides are potent suppressors of the growth of human tumor xenografts in nude mice. Oligonucleotides 16, 83-93. doi: 10.1089/oli.2006.16.83
Gokhale, P. C., Zhang, C., Newsome, J. T., Pei, J., Ahmad, I., Rahman, A., et al. (2002). Pharmacokinetics, toxicity, and efficacy of ends-modified raf antisense oligodeoxyribonucleotide encapsulated in a novel cationic liposome. Clin. Cancer Res. 8, 3611-3621.

Gomes, C. P., Ferreira Lopes, C. D., Duarte Moreno, P. M., Varela-Moreira, A., Alonso, M. J., and Pêgo, A. P. (2014). Translating chitosan to clinical delivery of nucleic acid-based drugs. MRS Bull. 39, 60-70. doi: 10.1557/mrs.2013.314

Graham, M. J., Crooke, S. T., Monteith, D. K., Cooper, S. R., Lemonidis, K. M., Stecker, K. K., et al. (1998). In vivo distribution and metabolism of a phosphorothioate oligonucleotide within rat liver after intravenous administration. J. Pharmacol. Exp. Ther. 286, 447-458.

Hammond, S. M., and Wood, M. J. (2011). Genetic therapies for RNA mis-splicing diseases. Trends Genet. 27, 196-205. doi: 10.1016/j.tig.2011.02.004

Henry, S. P., Templin, M. V., Gillett, N., Rojko, J., and Levin, A. A. (1999). Correlation of toxicity and pharmacokinetic properties of a phosphorothioate oligonucleotide designed to inhibit ICAM-1. Toxicol. Pathol. 27, 95-100. doi: 10.1177/019262339902700117

Henry, S., Stecker, K., Brooks, D., Monteith, D., Conklin, B., and Bennett, C. F. (2000). Chemically modified oligonucleotides exhibit decreased immune stimulation in mice. J. Pharmacol. Exp. Ther. 292, 468-479.

Hong, D. S., Kurzrock, R., Oh, Y., Wheler, J., Naing, A., Brail, L., et al. (2011). A phase 1 dose escalation, pharmacokinetic, and pharmacodynamic evaluation of eIF-4E antisense oligonucleotide LY2275796 in patients with advanced cancer. Clin. Cancer Res. 17, 6582-6591. doi: 10.1158/1078-0432.CCR-11-0430

Hovingh, K., Besseling, J., and Kastelein, J. (2013). Efficacy and safety of mipomersen sodium (Kynamro). Expert Opin. Drug Saf. 12, 569-579. doi: 10.1517/14740338.2013.793670

Iversen, P. L., Copple, B. L., and Tewary, H. K. (1995). Pharmacology and toxicology of phosphorothioate oligonucleotides in the mouse, rat, monkey and man. Toxicol. Lett. 82-83, 425-430. doi: 10.1016/0378-4274(95)03572-9

Jaaskelainen, I., Peltola, S., Honkakoski, P., Monkkonen, J., and Urtti, A. (2000). A lipid carrier with a membrane active component and a small complex size are required for efficient cellular delivery of anti-sense phosphorothioate oligonucleotides. Eur. J. Pharm. Sci. 10, 187-193. doi: 10.1016/S0928-0987(00)00068-3

Jain, R. K. (2012). Delivery of molecular and cellular medicine to solid tumors. $A d v$. Drug Deliv. Rev. 64, 353-365. doi: 10.1016/j.addr.2012.09.011

Jang, S. H., Wientjes, M. G., Lu, D., and Au, J. L. (2003). Drug delivery and transport to solid tumors. Pharm. Res. 20, 1337-1350. doi: 10.1023/A:1025785505977

Juliano, R., Bauman, J., Kang, H., and Ming, X. (2009). Biological barriers to therapy with antisense and siRNA oligonucleotides. Mol. Pharm. 6, 686-695. doi: $10.1021 / \mathrm{mp} 900093 \mathrm{r}$

Juliano, R. L., Ming, X., and Nakagawa, O. (2012). The chemistry and biology of oligonucleotide conjugates. Acc. Chem. Res. 45, 1067-1076. doi: 10.1021/ar2002123

Kawai, G., Yamamoto, Y., Kamimura, T., Masegi, T., Sekine, M., Hata, T., et al. (1992). Conformational rigidity of specific pyrimidine residues in tRNA arises from posttranscriptional modifications that enhance steric interaction between the base and the 2 '-hydroxyl group. Biochemistry 31, 1040-1046. doi: 10.1021/bi00119a012

Kibler-Herzog, L., Zon, G., Uznanski, B., Whittier, G., and Wilson, W. D. (1991). Duplex stabilities of phosphorothioate, methylphosphonate, and RNA analogs of two DNA 14-mers. Nucleic Acids Res. 19, 2979-2986. doi: 10.1093/nar/19.11.2979

Koshkin, A. A., Singh, S. K., Nielsen, P., Rajwanshi, V. K., Kumar, R., Meldgaard, M., et al. (1998). LNA (Locked Nucleic Acids): synthesis of the adenine, cytosine, guanine, 5-methylcytosine, thymine and uracil bicyclonucleoside monomers, oligomerisation, and unprecedented nucleic acid recognition. Tetrahedron 54, 3607-3630. doi: 10.1016/S0040-4020(98)00094-5

Krieg, A. M., Guga, P., and Stec, W. (2003). P-chirality-dependent immune activation by phosphorothioate $\mathrm{CpG}$ oligodeoxynucleotides. Oligonucleotides 13, 491-499. doi: 10.1089/154545703322860807

Krieg, A. M., and Stein, C. A. (1995). Phosphorothioate oligodeoxynucleotides: antisense or anti-protein? Antisense Res. Dev. 5, 241.

Manoharan, M. (1999). 2' -carbohydrate modifications in antisense oligonucleotide therapy: importance of conformation, configuration and conjugation. Biochim. Biophys. Acta 1489, 117-130. doi: 10.1016/S0167-4781(99)00138-4

Meidan, V. M., Glezer, J., Amariglio, N., Cohen, J. S., and Barenholz, Y. (2001). Oligonucleotide lipoplexes: the influence of oligonucleotide composition on 
complexation. Biochim. Biophys. Acta 1568, 177-182. doi: 10.1016/S03044165(01)00216-1

Minshull, J., and Hunt, T. (1986). The use of single-stranded DNA and RNase H to promote quantitative "hybrid arrest of translation" of mRNA/DNA hybrids in reticulocyte lysate cell-free translations. Nucleic Acids Res. 14, 6433-6451. doi: $10.1093 / \mathrm{nar} / 14.16 .6433$

Mocanu, J. D., Yip, K. W., Skliarenko, J., Shi, W., Busson, P., Lo, K. W., et al. (2007). Imaging and modulating antisense microdistribution in solid human xenograft tumor models. Clin. Cancer Res. 13, 5935-5941. doi: 10.1158/1078-0432.CCR06-3085

Monia, B. P., Lesnik, E. A., Gonzalez, C., Lima, W. F., McGee, D., Guinosso, C. J., et al. (1993). Evaluation of $2^{\prime}$-modified oligonucleotides containing $2^{\prime}$-deoxy gaps as antisense inhibitors of gene expression. J. Biol. Chem. 268, 14514-14522.

Mou, T. C., Gray, C. W., Terwilliger, T. C., and Gray, D. M. (2001). Ff gene 5 protein has a high binding affinity for single-stranded phosphorothioate DNA. Biochemistry 40, 2267-2275. doi: 10.1021/bi002136f

Nakamura, H., Oda, Y., Iwai, S., Inoue, H., Ohtsuka, E., Kanaya, S., et al. (1991). How does RNase H recognize a DNA.RNA hybrid? Proc. Natl. Acad. Sci. U.S.A. 88, 11535-11539. doi: 10.1073/pnas.88.24.11535

Netti, P. A., Berk, D. A., Swartz, M. A., Grodzinsky, A. J., and Jain, R. K. (2000). Role of extracellular matrix assembly in interstitial transport in solid tumors. Cancer Res. 60, 2497-2503.

Nielsen, P. E., Egholm, M., Berg, R. H., and Buchardt, O. (1991). Sequence-selective recognition of DNA by strand displacement with a thymine-substituted polyamide. Science 254, 1497-1500. doi: 10.1126/science. 1962210

Nishizaki, T., Iwai, S., Ohtsuka, E., and Nakamura, H. (1997). Solution structure of an RNA.2'-O-methylated RNA hybrid duplex containing an RNA.DNA hybrid segment at the center. Biochemistry 36, 2577-2585. doi: 10.1021/bi962297c

Obika, S., Nanbu, D., Hari, Y., Andoh, J.-I., Morio, K.-I., Doi, T., et al. (1998). Stability and structural features of the duplexes containing nucleoside analogues with a fixed N-type conformation, $2^{\prime}$-O $4^{\prime}$-C-methyleneribonucleosides. Tetrahedron Lett. 39, 5401-5404. doi: 10.1016/S0040-4039(98)01084-3

Pasternak, A., and Wengel, J. (2011). Unlocked nucleic acid-an RNA modification with broad potential. Org. Biomol. Chem. 9, 3591-3597. doi: 10.1039/c0ob01085e

Plenat, F., Klein-Monhoven, N., Marie, B., Vignaud, J. M., and Duprez, A. (1995). Cell and tissue distribution of synthetic oligonucleotides in healthy and tumor-bearing nude mice. An autoradiographic, immunohistological, and direct fluorescence microscopy study. Am. J. Pathol. 147, 124-135.

Senn, J. J., Burel, S., and Henry, S. P. (2005). Non-CpG-containing antisense 2 -methoxyethyl oligonucleotides activate a proinflammatory response independent of Toll-like receptor 9 or myeloid differentiation factor 88. J. Pharmacol. Exp. Ther. 314, 972-979. doi: 10.1124/jpet.105.084004

Seong, J. H., Lee, K. M., Kim, S. T., Jin, S. E., and Kim, C. K. (2006). Polyethylenimine-based antisense oligodeoxynucleotides of IL-4 suppress the production of IL-4 in a murine model of airway inflammation. J. Gene Med. 8, 314-323. doi: 10.1002/jgm.848

Simoes, S., Filipe, A., Faneca, H., Mano, M., Penacho, N., Duzgunes, N., et al. (2005). Cationic liposomes for gene delivery. Expert Opin. Drug Deliv. 2, 237-254. doi: 10.1517/17425247.2.2.237

Srinivasan, S. K., Tewary, H. K., and Iversen, P. L. (1995). Characterization of binding sites, extent of binding, and drug interactions of oligonucleotides with albumin. Antisense Res. Dev. 5, 131-139.

Stanton, R., Sciabola, S., Salatto, C., Weng, Y., Moshinsky, D., Little, J., et al. (2012). Chemical modification study of antisense gapmers. Nucleic Acid Ther. 22, 344-359. doi: 10.1089/nat.2012.0366

Stein, C. A., Hansen, J. B., Lai, J., Wu, S., Voskresenskiy, A., Hog, A., et al. (2010). Efficient gene silencing by delivery of locked nucleic acid antisense oligonucleotides, unassisted by transfection reagents. Nucleic Acids Res. 38, e3. doi: 10.1093/nar/gkp841

Stessl, M., Marchetti-Deschmann, M., Winkler, J., Lachmann, B., Allmaier, G., and Noe, C. R. (2009). A proteomic study reveals unspecific apoptosis induction and reduction of glycolytic enzymes by the phosphorothioate antisense oligonucleotide oblimersen in human melanoma cells. J. Proteomics 72, 1019-1030. doi: 10.1016/j.jprot.2009.06.001

Stewart, A. J., Pichon, C., Meunier, L., Midoux, P., Monsigny, M., and Roche, A. C. (1996). Enhanced biological activity of antisense oligonucleotides complexed with glycosylated poly-L-lysine. Mol. Pharmacol. 50, 1487-1494.

Straarup, E. M., Fisker, N., Hedtjarn, M., Lindholm, M. W., Rosenbohm, C., Aarup, V., et al. (2010). Short locked nucleic acid antisense oligonucleotides potently reduce apolipoprotein B mRNA and serum cholesterol in mice and non-human primates. Nucleic Acids Res. 38, 7100-7111. doi: 10.1093/nar/gkq457

Summerton, J., and Weller, D. (1997). Morpholino antisense oligomers: design, preparation, and properties. Antisense Nucleic Acid Drug Dev. 7, 187-195. doi: 10.1089/oli.1.1997.7.187

Van Vlerken, L. E., Vyas, T. K., and Amiji, M. M. (2007). Poly(ethylene glycol)modified nanocarriers for tumor-targeted and intracellular delivery. Pharm. Res. 24, 1405-1414. doi: 10.1007/s11095-007-9284-6

Watanabe, T. A., Geary, R. S., and Levin, A. A. (2006). Plasma protein binding of an antisense oligonucleotide targeting human ICAM-1 (ISIS 2302). Oligonucleotides 16, 169-180. doi: 10.1089/oli.2006.16.169

Watts, J. K., and Corey, D. R. (2012). Silencing disease genes in the laboratory and the clinic. J. Pathol. 226, 365-379. doi: 10.1002/path.2993

Webb, M. S., Tortora, N., Cremese, M., Kozlowska, H., Blaquiere, M., Devine, D. V., et al. (2001). Toxicity and toxicokinetics of a phosphorothioate oligonucleotide against the c-myc oncogene in cynomolgus monkeys. Antisense Nucleic Acid Drug Dev. 11, 155-163. doi: 10.1089/108729001300338681

Winkler, J., Stessl, M., Amartey, J., and Noe, C. R. (2010). Off-target effects related to the phosphorothioate modification of nucleic acids. Chem. Med. Chem. 5, 1344-1352. doi: 10.1002/cmdc.201000156

Wisse, E., Jacobs, F., Topal, B., Frederik, P., and De Geest, B. (2008). The size of endothelial fenestrae in human liver sinusoids: implications for hepatocytedirected gene transfer. Gene Ther. 15, 1193-1199. doi: 10.1038/gt.2008.60

Wu, X. A., Choi, C. H., Zhang, C., Hao, L., and Mirkin, C. A. (2014). Intracellular fate of spherical nucleic acid nanoparticle conjugates. J. Am. Chem. Soc. 136, 7726-7733. doi: 10.1021/ja503010a

Yin, H., Kanasty, R. L., Eltoukhy, A. A., Vegas, A. J., Dorkin, J. R., and Anderson, D. G. (2014). Non-viral vectors for gene-based therapy. Nat. Rev. Genet. 15, 541-555. doi: 10.1038/nrg3763

Zhang, C., Newsome, J. T., Mewani, R., Pei, J., Gokhale, P. C., and Kasid, U. N. (2009). Systemic delivery and pre-clinical evaluation of nanoparticles containing antisense oligonucleotides and siRNAs. Methods Mol. Biol. 480, 65-83. doi: 10.1007/978-1-59745-429-2_5

Zhang, Y., Qu, Z., Kim, S., Shi, V., Liao, B., Kraft, P., et al. (2011). Down-modulation of cancer targets using locked nucleic acid (LNA)-based antisense oligonucleotides without transfection. Gene Ther. 18, 326-333. doi: 10.1038/gt.2010.133

Conflict of Interest Statement: The authors declare that the research was conducted in the absence of any commercial or financial relationships that could be construed as a potential conflict of interest.

Received: 31 August 2014; paper pending published: 10 September 2014; accepted: 23 September 2014; published online: 14 October 2014.

Citation: Moreno PMD and Pêgo AP (2014) Therapeutic antisense oligonucleotides against cancer: hurdling to the clinic. Front. Chem. 2:87. doi: 10.3389/fchem. 2014.00087

This article was submitted to Chemical Engineering, a section of the journal Frontiers in Chemistry.

Copyright (C) 2014 Moreno and Pêgo. This is an open-access article distributed under the terms of the Creative Commons Attribution License (CC BY). The use, distribution or reproduction in other forums is permitted, provided the original author(s) or licensor are credited and that the original publication in this journal is cited, in accordance with accepted academic practice. No use, distribution or reproduction is permitted which does not comply with these terms. 